\title{
O lado de início da doença influencia a destreza manual de pacientes com doença de Parkinson
}

\author{
The onset side of the disease influences the manual dexterity in patients \\ with Parkinson's disease
}

\author{
Juliana Lahr ${ }^{1}$, Marcelo Pinto Pereira ${ }^{2}$, Paulo Henrique Silva Pelicioni ${ }^{3}$, Rosangela \\ Alice Batistela ${ }^{4}$, Lilian Teresa Bucken Gobbi ${ }^{5}$
}

doi: http://dx.doi.org/10.11606/issn.2238-6149.v29i3p223-229

Lahr J, Pereira MP, Pelicioni PHS, Batistela RA, Gobbi LTB. O lado de início da doença influencia a destreza manual de pacientes com doença de Parkinson. Rev Ter Ocup Univ Sao Paulo. 2018 set.-dez.;29(3):223-9.

RESUMO: A doença de Parkinson (DP) é caracterizada pelo início assimétrico de sintomas motores e compromete a destreza manual. Espera-se que pacientes com o lado de início da doença preferido comprometido consigam manter bom desempenho com o lado mais afetado (LMA) no teste de destreza manual decorrente da experiência motora ao longo da vida. O objetivo do estudo foi verificar a interferência da coincidência entre o lado de início da doença e preferência manual no desempenho da destreza manual, LMA e lado menos afetado, em pacientes com DP. Os pacientes foram distribuídos conforme o lado de início: Grupo Coincidente (lado preferido acometido) e Grupo Não Coincidente (lado não preferido acometido). A destreza manual foi avaliada pelo teste Annett Pegboard adaptado. A análise estatística ANOVA de dois fatores (grupo x lado), medidas repetidas no último fator, revelou que o Grupo Não Coincidente dispendeu maior tempo para completar o teste com o LMA ( $\mathrm{p}=0,001)$, enquanto, o Grupo Coincidente dispendeu o mesmo tempo com ambos os lados, indicando que pacientes nos estágios iniciais e com início da doença pelo lado preferido perdem a proficiência manual. Sugerese que intervenções para melhorar as habilidades manuais sejam aplicadas desde o diagnóstico da doença, principalmente em pacientes com o lado preferido acometido.

Descritores: Hipocinesia; Destreza motora; Mãos; Lateralidade funcional.
Lahr J, Pereira MP, Pelicioni PHS, Batistela RA, Gobbi LTB The onset side of the disease influences the manual dexterity in patients with Parkinson's disease. Rev Ter Ocup Univ Sao Paulo. 2018 Sept-Dec;29(3):223-9.

ABSTRACT: Parkinson's disease (PD) is characterized by the asymmetric onset of motor symptoms and manual dexterity. It is expected that patients with preferred onset side affected can maintain a good performance with the more affected side (MAS) due to the life-long motor experience. The aim of the study was to verify the interference of coincidence between the disease onset side and manual preference in the performance of manual dexterity, MAS and less affected side in patients with PD. The patients were distributed according to the disease onset side: Coincident group (preferred affected side) and non-coincident group (nonpreferred affected side). Manual dexterity was assessed by the Annett Pegboard adapted test. The statistical analysis ANOVA for two factors (group x side), with repeated measures in the last factor, revealed that the Non-coincident group spent more time to complete the test with the MAS ( $\mathrm{p}=0.001$ ), while the Coincident group spent the same time to perform the test with both sides, indicating that patients in the early stages of PD and disease onset by preferred side lose the manual proficiency. Therefore, interventions to improve manual skills have become important since the diagnosis of the disease, especially in patients with the preferred affected side.

Keywords: Hypokinesia; Motor skills; Hand; Functional laterality.

1. Mestre em Ciências da Motricidade pela Universidade Estadual Paulista - UNESP, Doutoranda do Programa de Pós-graduação em Ciências da Motricidade, Universidade Estadual Paulista - UNESP, Laboratório de Estudos da Postura e Locomoção. ORCID: http://orcid.org/0000-00018687-2771. E-mail: ju lahr@hotmail.com.

2. Doutor em Ciências da Motricidade pela Universidade Estadual Paulista - UNESP e Pós-doutorando do Programa de Pós-graduação em Ciências da Motricidade, Universidade Estadual Paulista - UNESP, Laboratório de Estudos da Postura e Locomoção. ORCID: http://orcid. org/0000-0002-9442-0385. E-mail: mppereir@yahoo.com.br.

3. Mestre em Ciências da Motricidade pela Universidade Estadual Paulista - UNESP, Doutorando em Public Health and Community Medicine pela University of New South Wales, Austrália. ORCID: http://orcid.org/0000-0003-3168-3388. E-mail: p.pelicioni@neura.edu.au.

4. Mestra em Ciências da Motricidade pela Universidade Estadual Paulista - UNESP, Doutoranda do Programa de Reabilitação e Desempenho Funcional da Faculdade de Medicina de Ribeirão Preto, Laboratório de Biomecânica e Controle Motor, Escola de Educação Física e Esporte de Ribeirão Preto. ORCID: http://orcid.org/0000-0003-3062-2209. E-mail: rosangela_batistela@yahoo.com.br.

5. Doutora em Kinesiology pela University of Waterloo - UW. Professor Adjunto da Universidade Estadual Paulista - UNESP, Laboratório de Estudos da Postura e Locomoção, Programa de Pós-graduação em Ciências da Motricidade, Universidade Estadual Paulista - UNESP. ORCID: http://orcid.org/0000-0003-3984-3403. E-mail: 1tbgobbi@rc.unesp.br.

Endereço para correspondência: Lilian Teresa Bucken Gobbi. Departamento de Educação Física, Instituto de Biociências, Universidade Estadual Paulista - UNESP. Avenida 24-A, 1515. Bairro Bela Vista. Rio Claro, SP, Brasil. CEP: 13506-900. 


\section{INTRODUÇÃO}

Aneurodegenerativa progressiva, caracterizada pelo início unilateral dos sintomas, decorrentes da perda assimétrica da dopamina nos núcleos da base ${ }^{1,2}$. A assimetria dos sintomas motores persiste ao longo do percurso da doença; assim, existirá um lado mais comprometido, aquele em que a doença se iniciou (LMA - lado mais afetado) e um lado que posteriormente será comprometido, porém sempre será menos afetado que o primeiro (LME - lado menos afetado) ${ }^{1}$.

O lado de início dos sintomas motores está associado à preferência manual ${ }^{3}$, sendo que a maior parte dos pacientes com DP apresenta o início da doença pelo lado de preferência manual ${ }^{2}$. Tal fato torna-se importante quando consideramos que a preferência lateral está frequentemente associada à vantagem de um dos lados do corpo em relação ao outro ${ }^{4}$. O comprometimento do lado preferido pode influenciar negativamente no desempenho das habilidades motoras 5 , como a destreza manual.

Uma das queixas mais frequentes dos pacientes com DP está justamente relacionada à destreza manual ${ }^{6}$, que é comprometida desde o estágio inicial da doença7 ${ }^{7}$. Esse comprometimento gera limitações no trabalho e no lazer ${ }^{8}$, dificulta a realização das atividades de vida diária, levando à redução da qualidade de vida e independência ${ }^{9}$, tais motivos justificaram e motivaram a realização deste estudo.

Apesar do lado de início da DP estar associado à preferência manual ${ }^{3}$, não tem sido investigado como o lado de início da doença poderia interferir no desempenho da destreza manual ${ }^{10}$. Os estudos têm avaliado o comprometimento da destreza manual segundo o LMA e LME $^{9,11}$, sem considerar se o LMA é o lado de preferência ou não.

Em virtude da característica assimétrica da DP é esperado pior desempenho do LMA quando uma tarefa manual é realizada ${ }^{11}$. Considerando que o comprometimento motor é menor no início da doença, espera-se que pacientes com lado de início preferido comprometido consigam manter um bom desempenho do LMA no teste de destreza manual, apesar das limitações impostas pela doença, tendo em vista a experiência motora ao longo da vida ${ }^{4}$ e pelo menor comprometimento motor (avaliado pela Unified Parkinson's Disease Rating Scale (UPDRS) - subescala 3) quando comparados a pacientes com o início pelo lado não preferido ${ }^{12}$, porém esta hipótese ainda não foi testada.

Um teste utilizado na clínica para avaliar a destreza manual é o teste Annett Pegboard. Este teste é de fácil aplicação e consiste em transferir e posicionar pinos com diferentes encaixes e diâmetros nos orifícios correspondentes ${ }^{13}$. O teste Annett Pegboard foi adaptado, apresentando como modificações a padronização do formato do encaixe e do diâmetro do pino, tais padronizações diminuem o componente cognitivo do teste, priorizando a avaliação do componente motor da destreza manual ${ }^{14}$. O teste Annett Pegboard adaptado já foi utilizado na DP, sendo capaz de discriminar diferenças relacionadas à severidade e ao subtipo da doença ${ }^{15}$.

O entendimento da interferência do lado de início da doença na destreza manual (avaliado pelo teste Annett Pegboard adaptado) poderia contribuir para esclarecimentos a respeito do comprometimento do LMA e LME em pacientes com DP, o que possibilitaria o desenvolvimento de melhores procedimentos de intervenção e prevenção com intuito de amenizar as perdas das habilidades manuais e funcionalidade desta população. Assim, o objetivo deste estudo foi verificar a interferência da coincidência entre o lado de início da doença e a preferência manual no desempenho da destreza manual (LMA e LME) em pacientes com DP.

\section{PROCEDIMENTOS METODOLÓGICOS}

\section{Participantes}

Os participantes deste estudo foram selecionados por conveniência junto ao Programa de Atividade Física para pacientes com doença de Parkinson (PROPARKI) da Universidade Estadual Paulista - UNESP - Campus Rio Claro. Participaram deste estudo 16 pacientes com DP idiopática, destros, sendo 8 pacientes com lado preferido acometido pela doença (Grupo Coincidente) e 8 pacientes com lado não preferido acometido (Grupo Não Coincidente); similares quanto a idade, tempo de doença, gênero, estágio e comprometimento da doença (Tabela 1). Os critérios de exclusão foram: a) pacientes que não se apresentassem nos estágios iniciais da doença, ou seja, estágios acima de 1,5 na Escala de estágios de incapacidade de Hoehn e Yahr (H\&Y), versão modificada $\left.{ }^{16} ; b\right)$ funções cognitivas não preservadas no Mini-Exame do Estado Mental (MEEM) ${ }^{17}$; c) comprometimentos osteomioarticulares nos membros superiores e d) comprometimentos visuais não corrigidos com o uso de óculos. Este estudo aderiu aos princípios éticos para pesquisa envolvendo seres humanos, conforme Resolução 466/2012 do Conselho Nacional de Saúde e foi aprovado pelo comitê de ética e pesquisa do Instituto de Biociências da Universidade Estadual Paulista - UNESP (Protocolo $\mathrm{n}^{\circ}$ 3936, 05/06/2012). Todos os participantes após ciência e concordância, assinaram o termo de consentimento livre e esclarecido. 
Lahr J, et al. O lado de início da doença influencia a destreza manual de pacientes. Rev Ter Ocup Univ Sao Paulo. 2018 set.-dez.;29(3):223-9.

Tabela 1 - Caracterização da amostra

\begin{tabular}{|c|c|c|c|c|}
\hline Variáveis & Grupo Coincidente & Grupo Não Coincidente & p-valor & Tamanho do efeito \\
\hline Preferência lateral & $\mathrm{D}(\mathrm{n}=8)$ & $\mathrm{D}(\mathrm{n}=8)$ & ---- & ---- \\
\hline Lado de início & $\mathrm{D}(\mathrm{n}=8)$ & $\mathrm{E}(\mathrm{n}=8)$ & ---- & ---- \\
\hline Gênero (F/M) & $5 / 3$ & $5 / 3$ & ---- & ---- \\
\hline H\&Y $(1 / 1,5)$ & $3 / 5$ & $2 / 6$ & --- & ---- \\
\hline Idade (anos) & $65,88 \pm 5,48$ & $66,50 \pm 5,07$ & 0,668 & $\mathrm{~d}=-0,117$ \\
\hline Tempo de doença (anos) & $5,25 \pm 2,54$ & $7,63 \pm 3,15$ & 0,315 & $\mathrm{~d}=0,259$ \\
\hline UPDRS I (pontos) & $2,13 \pm 1,35$ & $3,50 \pm 1,77$ & 0,096 & $\mathrm{r}=-0,416$ \\
\hline UPDRS II (pontos) & $10,00 \pm 4,40$ & $12,63 \pm 3,29$ & 0,246 & $\mathrm{r}=-0.290$ \\
\hline UPDRS III (pontos) & $20,63 \pm 8,87$ & $18,50 \pm 7,19$ & 0,793 & $\mathrm{r}=-0.065$ \\
\hline UPRDS total (pontos) & $32,75 \pm 12,71$ & $34,63 \pm 9,91$ & 1,000 & $\mathrm{r}=0.000$ \\
\hline MEEM (pontos) & $26,13 \pm 3,04$ & $28,75 \pm 1,38$ & 0,054 & $\mathrm{r}=-0.482$ \\
\hline
\end{tabular}

D - direito; E - esquerdo; F - feminino; M - masculino; H\&Y - estágio da doença; UPDRS - Unified Parkinson’s Disease Rating Scale: UPDRS I-subescala I (comprometimento psíquico); UPDRS II - subescala II (comprometimento funcional); UPDRS III - subescala III (comprometimento motor); UPDRS total - (comprometimento total da doença); MEEM - Mini-Exame do Estado Mental (desempenho no rastreio cognitivo).

\section{Procedimentos}

Os pacientes com DP foram avaliados no Laboratório de Estudos da Postura e Locomoção da Universidade Estadual Paulista - UNESP - Campus Rio Claro, sob efeito da medicação antiparkinsoniana (aproximadamente 1 hora após a ingestão da mesma), quanto a suas condições clínicas e cognitivas e, na sequência, foi avaliada a destreza manual.

$\mathrm{Na}$ avaliação clínica, os pacientes foram avaliados quanto ao comprometimento e ao estágio de evolução da doença por meio de duas escalas: 1) A UPDRS que avalia o comprometimento da doença e é dividida nas seguintes subescalas: UPDRS I (componentes cognitivos, comportamentais e do humor), UPDRS II (atividades da vida diária) e UPDRS III (funções motoras). Quanto maior a pontuação nestas subescalas, maior é o comprometimento psíquico, funcional e motor respectivamente ${ }^{18}$. 2) Escala de estágios de incapacidade de $\mathrm{H} \& \mathrm{Y}$, versão modificada, que estabelece o estágio de evolução da doença. Foram incluídos neste estudo pacientes no Estágio 1: doença unilateral e Estágio 1,5: envolvimento axial e unilatera ${ }^{16}$. Após a avaliação clínica foi realizado o rastreio cognitivo por meio do $\operatorname{MEEM}^{19}$, sendo considerada a escolaridade como nota de corte segundo proposto por Brucki et al. ${ }^{17}$.

Os pacientes foram classificados quanto ao lado de início da doença segundo o cálculo da diferença das pontuações entre o hemicorpo direito e esquerdo, nos itens 20 a 23, 25 e 26 da UPDRS III $^{20}$. Estes itens avaliam a presença e o nível de comprometimento dos sintomas motores de tremor, rigidez e bradicinesia dos membros superiores e inferiores. $\mathrm{O}$ hemicorpo com maior pontuação foi considerado como o lado que a doença se iniciou. A preferência manual foi definida como a mão utilizada para escrever ${ }^{21}$.

Para a avaliação da destreza manual foi utilizado o teste Annett Pegboard adaptado ${ }^{14,15}$. O equipamento utilizado para o teste (Figura 1) consiste em um dispositivo retangular de madeira com $32 \mathrm{~cm}$ de comprimento, $18 \mathrm{~cm}$ de largura e $5 \mathrm{~cm}$ de espessura. Posicionado a $1,5 \mathrm{~cm}$ da borda de cada lado do retângulo, existem duas fileiras de $15 \mathrm{~cm}$ de comprimento com 10 orifícios (distantes em 2 $\mathrm{cm}$ ) com 1,2 cm de diâmetro e 3,5 $\mathrm{cm}$ de profundidade. $\mathrm{Na}$ fileira distante do participante foram posicionados 10 pinos (1 por orifício) com $7 \mathrm{~cm}$ de altura e 1,0 cm de diâmetro ${ }^{13}$. 


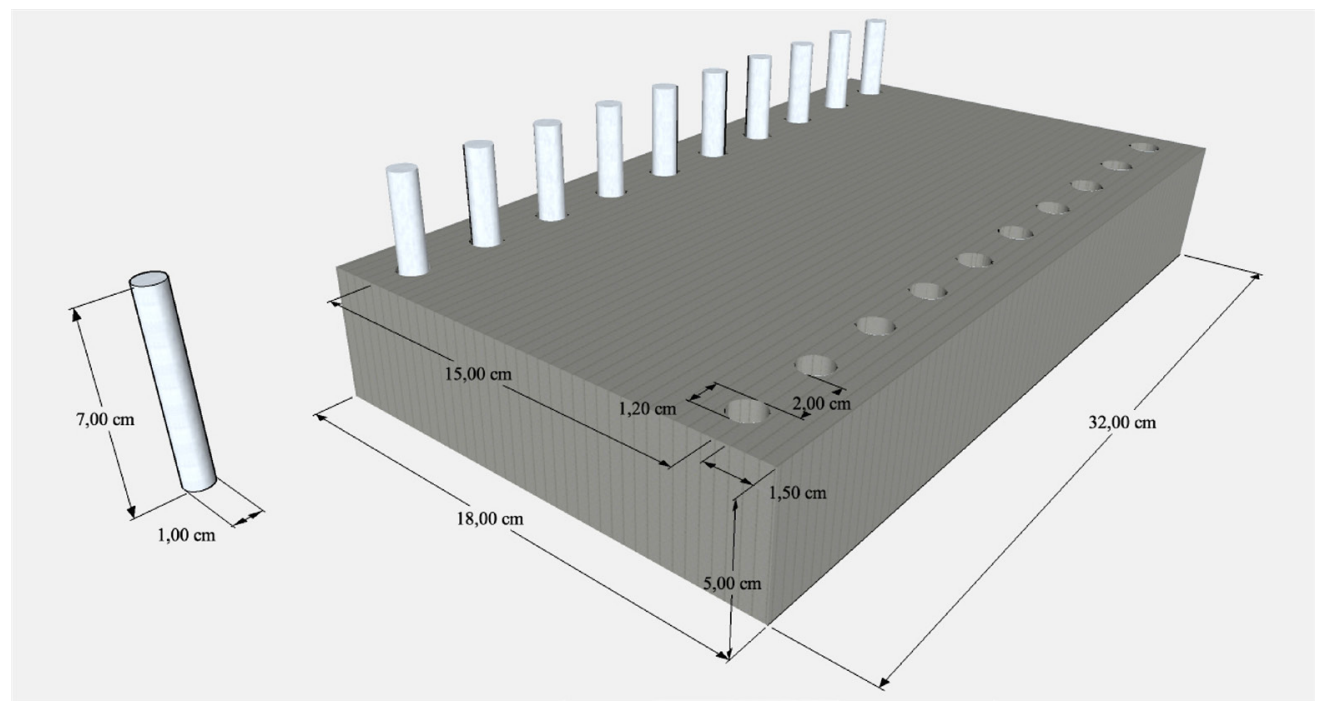

Figura 1 - Ilustração do Teste Annett Pegboard adaptado

Conforme descrição original do teste, o equipamento foi posicionado sobre uma mesa, de tal modo que a fileira com os pinos era a mais distante à frente do participante. O participante estava sentado e foi instruído a mover um pino por vez, o mais rápido possível colocando-os nos orifícios vazios correspondentes. $\mathrm{O}$ teste foi cronometrado e finalizado com a transferência de todos os pinos. O mesmo foi realizado com ambas as mãos: com a mão direita a movimentação dos pinos ocorreu da direita para a esquerda e vice-versa. Foi realizada uma tentativa de familiarização, que antecedeu três tentativas consecutivas de cada mão, sendo as três primeiras realizadas com a mão direita e as demais com a mão esquerda. Caso um pino caísse durante a tentativa, esta era repetida ${ }^{13}$. Durante o teste foi oferecido estímulo verbal, com a finalidade de otimizar o desempenho. Assim, quanto menor o tempo dispendido para a colocação dos pinos nos orifícios, melhor é considerada a destreza manual ${ }^{13,22}$. O tempo médio de três tentativas (em segundos) foi considerado para o LMA e para o LME.

\section{Análise estatística}

Inicialmente, a estatística descritiva foi empregada. Na sequência, a distribuição normal e a homogeneidade das variâncias foram observadas por meio dos testes de ShapiroWilk e Levene, respectivamente. Para a comparação entre grupos nas variáveis de caracterização foi utilizado o Teste $t$ de Student para amostras independentes, sendo calculado o tamanho do efeito por meio do d de Cohen $(d=\bar{x}-\bar{x} / \sigma)$.
Para as variáveis clínicas e cognitivas foi utilizado o Teste U de Mann-Whitney e os tamanhos do efeito calculados por meio do coeficiente de correlação $(\mathrm{r}=\mathrm{z} / \sqrt{\mathrm{N}})$. Para a comparação entre grupos (Grupo Coincidente x Grupo Não Coincidente) e lado avaliado (LMA x LME) no desempenho do teste de destreza manual foi utilizada a Análise de Variância (ANOVA) de dois fatores (grupo x lado) com medidas repetidas no último fator, sendo calculado o tamanho do efeito por meio do partial eta squared. $\mathrm{O}$ teste de post hoc de Bonferroni foi empregado quando interação entre fatores foi revelado. O programa SPSS (SPSS for Windows ${ }^{\circledR}$ - versão 21.0) foi utilizado para o tratamento estatístico e o nível de significância adotado foi de $\mathrm{p} \leq 0,05$.

\section{RESULTADOS}

O Test $t$ de Student e o teste U de Mann-Whitney não revelaram diferenças estatisticamente significativas na comparação entre os grupos nas variáveis de caracterização, clínicas e cognitiva (Tabela 1). A ANOVA revelou interação entre grupo e lado $\left(\mathrm{F}_{(2,15)}=11,10 ; \mathrm{p}=0,013 ; \eta_{p}^{2}=0,613\right)$. $\mathrm{O}$ teste de post hoc de Bonferroni revelou diferença significativa para o Grupo Não Coincidente, apresentando maior valor médio no tempo dispendido para completar o teste de destreza manual para o LMA em comparação com o LME ( $\mathrm{p}=0,001$ ), (Figura 2). Não foi identificada diferença para o Grupo Coincidente (LMA=LME; $\mathrm{p}=0,448$ ), que dispendeu o mesmo tempo para a realização da tarefa com ambas as mãos. 


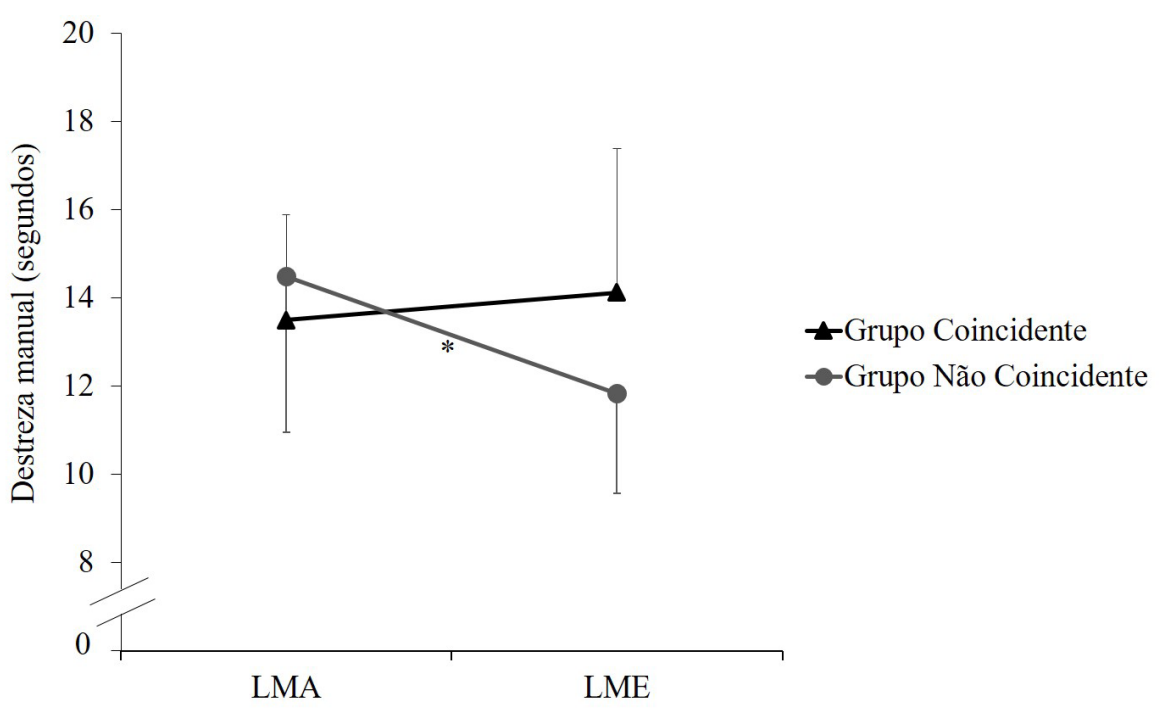

Legenda: LMA - Lado mais afetado; LME - Lado menos afetado; * Diferença estatisticamente significante para o Grupo Não Coincidente no teste de post hoc $(\mathrm{p}=0,001)$.

Figura 2 - Média e desvio-padrão do desempenho dos grupos no teste de destreza manual

\section{DISCUSSÃO}

O objetivo deste estudo foi verificar a interferência da coincidência entre o lado de início da doença e a preferência manual no desempenho da destreza manual (LMA e LME) em pacientes com DP. A nossa hipótese inicial foi que pacientes com início da doença pelo lado preferido (Grupo Coincidente) manteriam um bom desempenho do LMA no teste de destreza manual, quando comparados a pacientes com o início pelo lado não preferido (Grupo Não Coincidente), devido à experiência motora ao longo da vida e aos menores comprometimentos motores apresentados no início da doença. No entanto, nossos resultados não confirmam essa hipótese.

Os resultados demonstraram que pacientes que não coincidem o lado de início da doença com o lado preferido, dispendem mais tempo para completar uma tarefa de destreza manual com o LMA em relação ao LME, enquanto pacientes coincidentes dispendem o mesmo tempo para a realização da tarefa, independente do lado acometido. Estes resultados são discutidos quanto aos aspectos neuromotores, a complexidade da tarefa, os sintomas motores apresentados pelos pacientes e as implicações dos resultados para a prática clínica.

Ham et al. ${ }^{12}$ demonstraram que pacientes com início da doença pelo lado preferido apresentam menores comprometimentos motores (avaliados pela UPDRS III) quando comparados aos pacientes com acometimento no lado não preferido, embora ambos apresentem a mesma redução dopaminérgica estriatal. Esses autores sugerem que pacientes com início da doença pelo lado preferido apresentam redes motoras mais eficientes e com maior reserva neural no hemisfério cerebral preferido, permitindo assim, um bom desempenho motor e maior repertório cognitivo para superar melhor as alterações patológicas relacionadas à DP. No presente estudo, não foi observada a superioridade de desempenho do LMA preferido na destreza manual.

Estudos anteriores não investigaram o efeito da coincidência do lado de início da doença e da preferência manual no desempenho da destreza manual. Desta forma, podemos especular que a falta de superioridade no desempenho manual do LMA de pacientes coincidentes, seja decorrente da complexidade da tarefa. A tarefa de destreza manual requerer o controle coordenado de movimentos finos dos dedos e demanda de um processamento neural complexo ${ }^{23}$, complexidade esta que não seria suprida pelas redes motoras mais eficientes ${ }^{12}$. Portanto, o comprometimento da destreza manual parece ser mais impactante que outros comprometimentos no paciente com DP, principalmente quando o lado preferido é acometido.

Outros autores demonstraram que o membro superior preferido está predominantemente suscetível ao aparecimento de vários sintomas motores da doença, como bradicinesia e tremor ${ }^{3,24}$. A progressão da doença também dificulta o aprendizado de estratégias compensatórias para se 
ajustar aos comprometimentos motores ${ }^{25}$. Esta dificuldade de aprendizagem pode justificar os resultados encontrados, já que não foi observada estratégia compensatória para o LMA em pacientes coincidentes, mesmo nos estágios iniciais da doença. A não observância de estratégias compensatórias indica a perda da proficiência da mão preferida.

Nossos resultados evidenciam a importância de se considerar o lado de início da doença, preferido ou não, na avaliação entre membros em tarefas manuais. Sabe-se que nos estágios mais avançados da doença, os pacientes tendem a mudar a sua preferência manual ${ }^{10}$. Porém, o presente estudo demonstrou que desde os estágios iniciais da doença, os pacientes com início da doença pelo lado preferido não apresentam vantagens no desempenho do lado preferido. Diante destes resultados, tornam-se importantes intervenções voltadas para melhora/manutenção das habilidades manuais desde o diagnóstico inicial da doença, especialmente em pacientes com o lado preferido acometido. Além disso, o fato da destreza manual não ser responsiva à suplementação dopaminérgica e sua íntima relação com o desempenho na realização das atividades de vida diária reforçam a necessidade de intervenções específicas voltadas para essas habilidades manuais ${ }^{6}$.

Dentre as intervenções não-farmacológicas, para a recuperação e manutenção das habilidades manuais, destacam-se as intervenções da fisioterapia e da terapia ocupacional. Estas intervenções devem abordar principalmente tarefas que envolvam destreza manual utilizando-se técnicas adaptativas para minimizar as limitações decorrentes da progressão da doença e, consequentemente, promover melhora e manutenção da qualidade de vida nessa população ${ }^{7}$. Assim, para tais intervenções, torna-se imprescindível levar em consideração o desempenho no teste de destreza manual, como importante medidor das atividades de vida diária9.

O presente estudo apresenta como limitações o baixo número amostral e a falta de validação do instrumento utilizado. Sugere-se que os próximos estudos avaliem um maior número amostral, além de investigar os demais estágios da doença, moderado e avançado, para que se compreenda como o lado de início da doença influencia o desempenho da destreza manual ao longo da progressão da doença.

\section{CONCLUSÕES}

O lado de início da doença influenciou o desempenho da destreza manual, LMA e LME, de pacientes com DP. Pacientes que apresentaram início da doença pelo lado preferido mostraram déficits na proficiência da mão preferida, enquanto que pacientes com o lado não preferido acometido mantiveram bom desempenho com a mão preferida. Portanto, sugere-se que procedimentos de intervenção voltados para melhora e manutenção das habilidades manuais sejam realizados desde o diagnóstico inicial da doença, especialmente em pacientes com o lado preferido acometido, visando a manutenção da independência funcional.

Agradecimentos: os autores agradecem à Coordenação de Aperfeiçoamento de Pessoal de Nível Superior - Brasil (CAPES) - Código de Financiamento 001 e à Agência de Fomento Conselho Nacional de Desenvolvimento Científico e Tecnológico (CNPq) - Processos 306389/2013-4 e 142057/2017-7.

O artigo faz parte do projeto de pesquisa "Efeitos de um programa de atividade física sobre a condição clínica, o estado mental, a capacidade funcional e o comportamento postural e locomotor de idosos com Doença de Parkinson" que atendeu os procedimentos éticos vigentes. Parecer de comitê de ética institucional: aprovado CEP 3936 em 05 de junho de 2012. Apresentado como pôster "Lado de início da doença e desempenho no teste de destreza manual em indivíduos com doença de Parkinson” no VIII Congresso Internacional de Educação Física e Motricidade Humana - XIV Simpósio Paulista de Educação Física, Rio Claro, 2013.

Participação dos autores: Juliana Lahr - organização e análise dos dados, concepção e redação do texto; Paulo Henrique Silva Pelicioni e Rosangela Alice Batistela - coleta dos dados e a revisão do texto; Marcelo Pinto Pereira - processamento dos dados e pela concepção e revisão do texto; Lilian Teresa Bucken Gobbi - orientadora e responsável pela concepção do projeto e revisão do texto.

\section{REFERÊNCIAS}

1. Djaldetti R, Ziv I, Melamed E. The mystery of motor asymmetry in Parkinson's disease. Lancet Neurol. 2006;5(9):796-802. doi: $10.1016 / \mathrm{S} 1474-4422(06) 70549-\mathrm{X}$.

2. van der Hoorn A, Burger H, Leenders KL, de Jong BM. Handedness correlates with the dominant Parkinson side: a systematic review and meta-analysis. Mov Disord. 2012;27(2):206-10. doi: 10.1002/mds.24007.
3. Barrett MJ, Wylie SA, Harrison MB, Wooten GF. Handedness and motor symptom asymmetry in Parkinson's disease. J Neurol Neurosurg Psychiatr. 2011;82(10):1122-4. doi: 10.1136/jnnp.2010.209783.

4. Teixeira LA, Paroli R. Assimetrias laterais em ações motoras: preferência versus desempenho Motriz. Rev Educ Fis. 2000;6(1):1-8. Disponível em: http://www.rc.unesp.br/ib/ 
efisica/motriz/06n1/6n1Teixeira.pdf.

5. Brown SG, Roy EA, Rohr LE, Bryden PJ. Using hand performance measures to predict handedness. Laterality. 2006;11(1):1-14. doi: 10.1080/1357650054200000440.

6. Gebhardt A, Vanbellingen T, Baronti F, Kersten B, Bohlhalter S. Poor dopaminergic response of impaired dexterity in Parkinson's disease: bradykinesia or limb kinetic apraxia? Mov Disord. 2008;23(12):1701-6. doi: 10.1002/mds.22199.

7. Quinn L, Busse M, Dal Bello-Haas V. Management of upper extremity dysfunction in people with Parkinson disease and Huntington disease: facilitating outcomes across the disease lifespan. J Hand Ther. 2013;26(2):148-54. doi: 10.1016/j. jht.2012.11.001.

8. Jasinska-Myga B, Heckman MG, Wider C, Putzke JD, Wszolek ZK, Uitti RJ. Loss of ability to work and ability to live independently in Parkinson's disease. Parkinsonism Relat Disord. 2012;18(2):130-5. doi: 10.1016/j. parkreldis.2011.08.022.

9. Choi YI, Song CS, Chun BY. Activities of daily living and manual hand dexterity in persons with idiopathic Parkinson disease. J Phys Ther Sci. 2017;29(3):457-60. doi: 10.1589/ jpts.29.457.

10. Štochl, J, Hagtvet KA, Brozová H, Klempír J, Roth J, Růzicka E. Handedness does not predict side of onset of motor symptoms in Parkinson's disease. Mov Disord. 2009;24(12):1836-9. doi: 10.1002/mds.22653.

11. Haaxma CA, Helmich RC, Borm GF , Kappelle AC, Horstink MW , Bloem BR. Side of symptom onset affects motor dysfunction in Parkinson's disease. Neuroscience 2010;170(4):1282-5. doi: 10.1016/j.neuroscience.2010.07.030.

12. Ham JH, Lee JJ, Kim JS, Lee PH, Sohn YH. Is dominantside onset associated with a better motor compensation in Parkinson's disease? Mov Disord. 2015;30(14):1921-5. doi: $10.1002 / \mathrm{mds} .26418$.

13. Bryden PJ. The origins of manual asymmetries: what is revealed by pushing the limits of task difficulty [Tese]. Ontário/ Canadá: Universidade de Waterloo; 1998.

14. Teixeira-Arroyo C, Santos PCR, Gobbi LTB. Programa de atividade física para pacientes com doença de Parkinson: PROPARKI. In: Coelho FGM, Gobbi S, Costa JLR; Gobbi LTB, organizadores. Exercício físico no envelhecimento saudável e patológico: da teoria à prática. Curitiba: CRV; 2013. p.341-68.
15. Pereira MP, Pelicioni PHS, Gobbi LTB. Parkinson's disease severity and motor subtype influence physical capacity components. Motriz: Rev Educ Fis. 2013;19(3):605-13. https:// dx.doi.org/10.1590/S1980-65742013000300011.

16. Schenkman M, Wei Zhu C, Cutson TM, Whetten-Goldstein K. Longitudinal evaluation of economic and physical impact of Parkinson's disease. Parkinsonism Relat Disord. 2001;8(1):4150. doi: 10.1016/S1353-8020(00)00079-1.

17. Brucki SMD, Nitrini R, Caramelli P, Bertolucci PHF, Okamoto IH. Sugestões para o uso do Mini-exame do Estado Mental no Brasil. Arq Neuropsiquiatr 2003;61(3-B):777-81. doi: 10.1590/S0004-282X2003000500014.

18. Fahn S, Elton R, Members of the UPDRS Development Committee. Unified Parkinson's disease rating scale. In: Fahn S, Marsden CD, Calne DB, Goldstein M, eds. Recent developments in Parkinson's disease. v. 2. Florham Park, NJ: Macmillan Healthcare Information; 1987. p.153-63.

19. Folstein MF, Folstein SE, Mchugh PR. Minimental state: a practical method for grading the cognitive state of patients for the clinician. J Psychiatr Res. 1975;12(3):189-98. doi: 10.1016/0022-3956(75)90026-6.

20. Uitti RJ, Baba Y, Wszolek ZK, Putzke DJ. Defining the Parkinson's disease phenotype: initial symptoms and baseline characteristics in a clinical cohort. Parkinsonism Relat Disord. 2005;11(3):139-45. doi: 10.1016/j.parkreldis.2004.10.007.

21. Yust-Katz S, Tesler D, Treves TA, Melamed E, Djaldetti R. Handedness as a predictor of side of onset of Parkinson's disease. Parkinsonism Relat Disord. 2008;14(8):633-5. doi: 10.1016/j.parkreldis.2008.01.017.

22. Hamilton LD, Thomas E, Almuklass AM, Enoka RM. A framework for identifying the adaptations responsible for differences in pegboard times between middle-aged and older adults. Exp Gerontol. 2017;97:9-16. doi: 10.1016/j. exger.2017.07.003.

23. Perez MA. Neural control of hand movements. Motor Control. 2015;19(2):135-41. doi: 10.1123/mc.2014-0055.

24. Shi J, Liu J, Qu Q, et al. Handedness and dominant side of symptoms in Parkinson's disease. Med Clin (Barc). 2014;142(4):141-4. doi: 10.1016/j.medcli.2012.11.028.

25. Nieuwboer A, Rochester L, Müncks L, Swinnen SP. Motor learning in Parkinson's disease: limitations and potential for rehabilitation. Parkinsonism Relat Disord. 2009;15(Suppl 3):S53-8. doi: 10.1016/S1353-8020(09)70781-3. 